\title{
REVIEW
}

\section{MYASTHENIA GRAVIS AND COVID-19: A SYSTEMATIC REVIEW OF CASE REPORTS AND CASE SERIES}

\author{
Débora Lilian Roveron, Ivan Luiz Gonçalves dos Santos, Julio Luiz Gonçalves \\ dos Santos, Nájila Fernandes Alem and João Gabriel Pacetti Capobianco
}

\section{ABSTRACT}

Myasthenia gravis (MG) is an autoimmune disease involving neuromuscular transmission and possible respiratory failure when concomitant with COVID-19. The aim of this study was to analyze the need for ventilatory support (VS), length of hospital stay (LOS) and mortality in patients diagnosed with MG and COVID-19. In this systematic review, PubMed, SciELO, LILACS, MEDLINE and IBECS databases were searched for primary studies published from January 2010 to March 2021, with no language restrictions. Fourteen eligible studies were identified. The main factor associated with the need for VS was the use of antibiotics other than azithromycin (AZM) for the treatment of COVID-19 (RR 1.60; 95\% CI 1.20-2.91; $\mathrm{p}=0.009$ ). Patients who used hydroxychloroquine (HCQ) and AZM had almost twice the risk of needing invasive ventilatory support (IVS) (RR $1.94 ; 95 \%$ CI 1.07-3.52; $\mathrm{p}=0.16$ ). There were nonsignificant trends towards less need for IVS in patients who used intravenous immunoglobulin (IVIg) and corticosteroid therapy (RR $0.54 ; 95 \%$ CI $0.09-3.26 ; \mathrm{p}=0.60$ ). There was a trend towards shorter LOS in patients who received therapy with IVIg and corticosteroid therapy [8 (5 - 8) vs $19(12.2-23.7) ; \mathrm{p}=0.007] .10 .3 \%(\mathrm{n}=4 / 39)$ died and $100 \%$ did not use IVIg or IVIg and prednisone. There was a non-significant trend towards higher mortality in patients who used AZM (RR 2.55; 95\% CI 0.26-30.02; $\mathrm{p}=0.60$ ). IVIg and corticotherapy presented themselves as a favorable alternative in relation to the outcomes.

KEY WORDS: Coronavirus infections; length of stay; Myasthenia gravis; Respiratory insufficiency.

\section{INTRODUCTION}

COVID-19, a pandemic disease in 2020, is a respiratory infection generated by the SARS-CoV-2 virus, causing a severe acute respiratory syndrome. In March, according to Johns Hopkins University \& Medicine (2021), the

Pontifícia Universidade Católica de Minas Gerais, ICBS, Departamento de Medicina, Poços de Caldas, Minas Gerais, Brazil.

Débora Lilian Roveron: https://orcid.org/0000-0003-0740-1790; Ivan Luiz Gonçalves dos Santos: https://orcid.org/0000-00034203-5917; Julio Luiz Gonçalves dos Santos: https://orcid.org/0000-0002-8954-3129; Nájila Fernandes Alem: https://orcid. org/0000-0002-4054-3857; João Gabriel Pacetti Capobianco: https://orcid.org/0000-0003-2626-3880

Corresponding author: Débora Lilian Roveron, Av. Padre Cletus Francis Cox 1661, CEP 37714-620 Poços de Caldas, MG, Brazil. Email: deboraroveron@hotmail.com 
mortality rate for Sars-Cov-2 ranged from 0\% (Singapore) to $21.0 \%$ (Yemen), depending on the location. According to Ioannidis (2020), the global mortality rate was at $0.23 \%$, with a higher prevalence among the elderly patients with underlying comorbidities, and presenting immunological deficiencies (Aksoy \& Oztutgan, 2020; Keller et al., 2020).

Coronavirus 2 generates a massive inflammatory response, with lesions in skeletal and cerebrovascular muscles, in addition to neurological impairment. Symptoms may include fever, cough, diarrhea, dyspnea, hypoxia, hyposmia, myalgia, fatigue, headache, dysphagia, rhinorrhea, arthralgia, confusion, and hemoptysis. Severe cases may evolve to respiratory failure, requiring invasive (IVS) or non-invasive ventilatory support (NIVS) (Anand et al., 2020; Singh \& Govindarajan, 2020).

Myasthenia gravis (MG) is an autoimmune disease characterized by variable muscle weakness, fluctuating character, and potentially deleterious symptoms, such as respiratory failure. Neuromuscular transmission is compromised by autoantibodies linked to acetylcholine receptors or functional molecules, at the neuromuscular junction, affecting ocular, respiratory, bulbar and limb muscles. Treatment may include cholinesterase inhibitory drugs, such as pyridostigmine, immunosuppressants, such as prednisone and azathioprine, and immunomodulators, such as mycophenolate mofetil (MMF) (Hübers et al., 2020; Ruiter et al., 2020).

Symptoms of MG can worsen quickly and may be triggered by infectious conditions. This exacerbation may progress to restrictive respiratory failure, characterizing a myasthenic crisis. Plasmapheresis (PLEX) and intravenous immunoglobulin (IVIg) are essential for successful treatment during myasthenic crises or respiratory complications on worsening or life risk are detected (Jaretzki et al., 2000; Conitec, 2020; Ramaswamy \& Govindarajan, 2020).

The use of hydroxychloroquine (HCQ) and chloroquine (CQ) can cause myasthenia gravis, as has been known since 1981 (Schumm et al., 1981). Likewise, AZM may trigger myasthenic crises (Anand et al., 2020). Such drugs are still prescribed indiscriminately by doctors who judge the benefits to be greater than the harms. In both MG and COVID-19, drug suppression in immune and inflammatory processes can be simultaneous (Ruiter et al., 2020).

There is no high-quality scientific evidence to guide the care management of patients with MG infected by Sars-Cov-2, according to their risks and particularities, despite the existence of expert opinions. With regard to public health relevance, the determination of risk factors that may aggravate underlying diseases is urgent. These may be MG, leading to unnecessary longer hospitalization, since the cure of the most serious cases is related to availability of resources in the health field, such as workforce, beds in intensive care units (ICUs) and mechanical ventilators (Emanuel et al., 2020; Remuzzi \& Remuzzi, 2020). 
This study aims to analyze scientific evidence on the management, evolution, and prognosis of patients with MG and COVID-19, to clarify and fill the knowledge gaps in the presentation of both diseases. The PICO strategy (Patient, Intervention, Comparison and Outcomes) (Santos et al., 2007) was used to elaborate the guiding question: "Do patients with myasthenia gravis infected by Sars-Cov-2, have a greater chance of hospitalization, need for ventilatory support and mortality if treated with azithromycin and/or hydroxychloroquine compared to intravenous immunoglobulin and prednisone?"

\section{METHODS}

The eligibility criteria were primary studies on human beings, published from January 2010 to March 2021, with no language restrictions. Inclusion criteria were patients previously diagnosed with myasthenia gravis, with no restriction of genre, age group or location, and infected with Sars-Cov-2. The exclusion criteria were studies on patients who had hydroxychloroquineinduced or diagnosed myasthenia gravis after Sars-Cov-2 infection.

The search strategy used was: ("Myasthenia gravis" or "Myasthenia serious") and ("COVID"). Case series, case reports (non-comparative observational studies) and retrospective cross-sectional studies were included through PubMed (64 articles) and SciELO (1 article) databases. The articles were initially selected by title and abstract, and then added to the review after applying inclusion and exclusion criteria.

The variables analyzed were gender (as a biological factor), age, treatment with IVIg and prednisone without HCQ and AZM, use of AZM and use of HCQ. The analyzed outcomes were need for ventilatory support (VS), mean hospital stay (LOS) and mortality. Two independent researchers evaluated the methodological quality, risk of bias and level of evidence of this systematic review, using the AMSTAR-2 (Assessing the Methodological Quality of Systematic Reviews 2), PRISMA (Preferred Reporting Items for Systematic Reviews and Meta-Analyses) (Moher et al., 2009) recommendation and GRADE (Grading of Recommendations Assessment, Development and Evaluation). This systematic review was registered in PROSPERO under number CRD42021241404.

\section{Statistical analysis}

Categorical variables were described as frequencies and percentages and tested using the chi-square test or Fisher's exact test. The relationships between categorical variables were described using the relative risk (RR) as a measure of association, with $95 \%$ confidence intervals $(95 \% \mathrm{CI})$. Continuous variables with normal distribution were described as mean and standard deviation (SD), and non-normal variables as median and interquartile range (IIQ). Normal 
continuous variables were tested with the Student's t test for independent samples, and non-normal continuous variables with the Mann-Whitney U test. $\mathrm{p}<0.05$ was considered statistically significant. The analysis were performed using the statistical program Statistical Package for the Social Sciences (SPSS version 20.0).

\section{RESULTS}

The search identified 65 articles, of which 14 were selected for the final sample, according to the following eligibility criteria: non-comparative observational studies and myasthenia gravis not caused by Sars-Cov-2 and hydroxychloroquine (Figure 1). As Camelo-Filho et al. (2020) did not determine the gender and age of its participants, this study was not considered for the final calculation of the percentage of these variables.
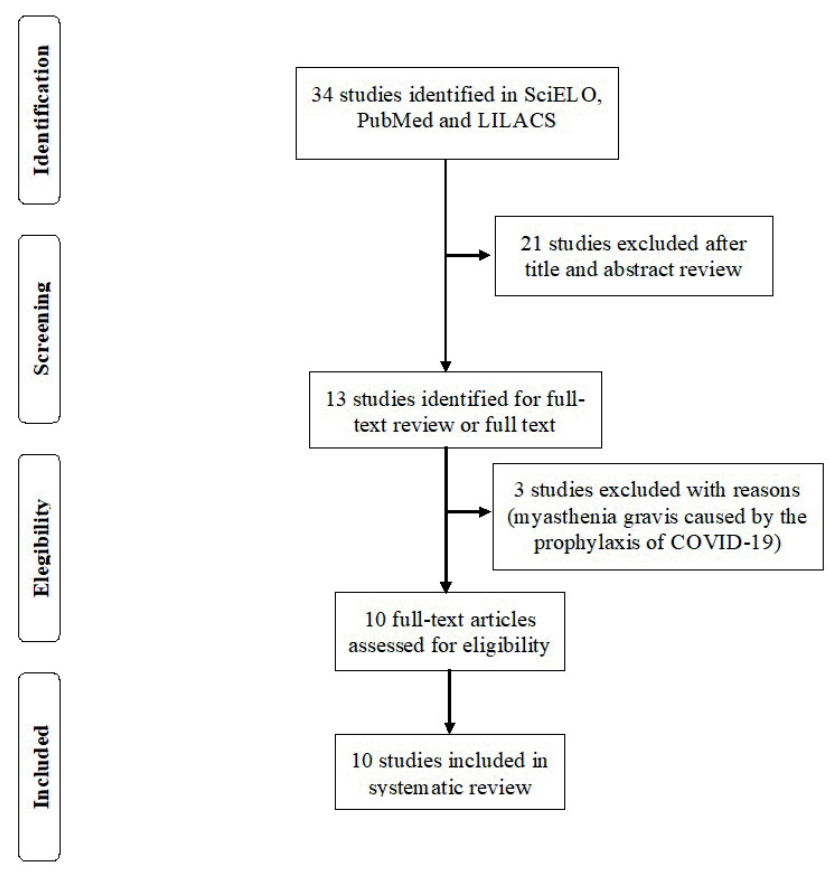

Figure. Study selection. 
Ventilatory support was performed in 26 patients $(66 \%)$ in the sample with $18(46 \%)$ needing IVS. The main factor associated with the need for SV was the use of antibiotics other than AZM for the treatment of COVID-19 (RR $1.60 ; 95 \%$ CI $1.20-2.91 ; \mathrm{p}=0.009)$, and there was also a non-significant trend towards greater IVS (RR 1.34; 95\% CI 0.66-2.73; $\mathrm{p}=0.39$ ). Patients who used HCQ and AZM had almost twice the risk of needing invasive ventilatory support (IVS) (RR 1.94; 95\% CI 1.07-3.52; $\mathrm{p}=0.16$ ). In addition, we highlight the non-significant trends for lower need for IVS in patients who underwent therapy with IVIg (RR 0.86; 95\% CI 0.19-3.03; $\mathrm{p}=0.62$ ) and in patients who used IVIg associated with corticosteroid therapy (RR 0.57; 95\% CI $0.16-1.93$; $\mathrm{p}=0.60$ ).

Most of the cases found required hospitalization $(79 \%, n=31 / 39)$, with an average hospital stay of 15 days (IIQ 9-22.5). The main factors associated with the length of hospital stay are shown in Table 1. The use of other antibiotics in addition to azithromycin for the treatment of COVID-19 was associated with an increase in LOS [20 days (9.5-32) vs 10 days (9-14); $p=0.01]$. The main factor associated with reduced hospital stay was use of IVIg associated with corticosteroid therapy [8 (5-8) vs $19(12.2-23.7) ; p=0.007]$. In addition, there was a statistically non-significant trend towards shorter hospital stays in patients who used only IVIg [9 (6.5-40) vs 19 (13.75-21.75); $p=0.12$ ].

In the sample of cases found in this review, 4 deaths were reported in patients with myasthenia gravis infected with SARS-CoV-2, resulting in a mortality rate of $10.3 \%(n=4 / 39)$. Although no factor was associated in a statistically significant way with mortality, there was a non-significant trend towards higher mortality in patients who used AZM (RR 2.55; 95\% CI 0.26$30.02 ; \mathrm{p}=0.60$ ), and in patients who used antibiotics other than azithromycin (RR 2.5; 95\% CI 0.23-26.6; $\mathrm{p}=0.61$ ). In addition, all patients who died did not use IVIg or IVIg associated with prednisone (Table 2).

The treatment variables for MG and COVID-19, in addition to the analyzed outcomes of the total sample, are shown in Table 3 . The sample was coded in increasing numerical order, according to the authors' alphabetical order. The variables and results analyzed for the thirty-nine patients are shown in Tables 1, 2, 4 and 5. The analysis of authors, year of publication, funding and location of the studies included in this review are shown in Table 6.

The AMSTAR-2 consists of 16 items evaluated as "yes", "no" or "partial yes", which measure the quality of the systematic review in "high," "moderate", "low" or "critically low" (Shea et al., 2017). This study received "moderate" quality. Through the GRADE (Grading of Recommendations Assessment, Development and Evaluation), the articles included in this systematic review are classified as low quality of evidence because they are non-comparative observational studies, difficult to reproduce in experimental studies. 
Table 1. Factors associated with length of hospital stay.

\begin{tabular}{|c|c|c|}
\hline Variables & $\begin{array}{l}\text { Length of hospital stay } \\
\text { (days) }\end{array}$ & $\mathrm{p}$ value \\
\hline \multicolumn{3}{|c|}{ Sex, median (IIQ) } \\
\hline Masculine & $14(9-63)$ & $0.21^{\mathrm{a}}$ \\
\hline Feminine & $10(5-17)$ & \\
\hline \multicolumn{3}{|c|}{ HCQ use, median (IIQ) } \\
\hline Yes & $17(11-20.75)$ & $0.61^{\mathrm{a}}$ \\
\hline No & $13.5(8.75-25)$ & \\
\hline \multicolumn{3}{|c|}{ AZM use, median (IIQ) } \\
\hline Yes & $14(9-27)$ & $0.96^{\mathrm{a}}$ \\
\hline No & $16.5(9-21.25)$ & \\
\hline \multicolumn{3}{|c|}{ HCQ + AZM use, median (IIQ) } \\
\hline Yes & $17(11-305)$ & $0.66^{\mathrm{a}}$ \\
\hline No & $14(9-22.5)$ & \\
\hline \multicolumn{3}{|c|}{$\begin{array}{l}\text { Use of other antibiotics for } \\
\text { COVID-19, median (IIQ) }\end{array}$} \\
\hline Yes & $20(9.5-32)$ & $0.01^{\mathrm{a}}$ \\
\hline No & $10(9-14)$ & \\
\hline \multicolumn{3}{|c|}{$\begin{array}{l}\text { IV Immunoglobulins use (without } \\
\text { HCQ or AZM), median (IIQ) }(n=13)\end{array}$} \\
\hline Yes & $9(6.5-40)$ & $0.12^{\mathrm{a}}$ \\
\hline No & $19(13.75-21.75)$ & \\
\hline \multicolumn{3}{|c|}{$\begin{array}{l}\text { IV Immunoglobulins and } \\
\text { corticotherapy use (without HCQ or } \\
\text { AZM), median (IIQ) }(n=13)\end{array}$} \\
\hline Yes & $8(5.0-8.0)$ & $0.007^{\mathrm{a}}$ \\
\hline No & $19(12.2-23.7)$ & \\
\hline
\end{tabular}

${ }^{\mathrm{a}}$ Mann Whitney U Test. Legend: AZM = azithromycin, HCQ = hydroxychloroquine, $\mathrm{IV}=$ intravenous. 
Table 2. Factors associated with mortality in patients with myasthenia gravis infected by SARS-CoV-2.

\begin{tabular}{|c|c|c|c|c|}
\hline Variables & $\begin{array}{l}\text { Death, } \\
\text { yes } \\
(n=4)\end{array}$ & $\begin{array}{c}\text { Death, } \\
\text { no } \\
(\mathrm{n}=33)\end{array}$ & RR (CI 95\%) & P Value \\
\hline \multicolumn{5}{|c|}{ HCQ use, n (\%) } \\
\hline Yes & $0(0)$ & $8(100)$ & - & $0.55^{\mathrm{a}}$ \\
\hline No & $4(13.8)$ & $25(86.2)$ & & \\
\hline \multicolumn{5}{|c|}{ AZM use, n (\%) } \\
\hline Yes & $3(15)$ & $17(85)$ & $2.55(0.26-30.02)$ & $0.60^{\mathrm{a}}$ \\
\hline No & $1(5.9)$ & $16(94.1)$ & 1 & \\
\hline \multicolumn{5}{|c|}{ HQC + AZM use, $n(\%)$} \\
\hline Yes & $0(0)$ & $4(100)$ & - & $1.0^{\mathrm{a}}$ \\
\hline No & $4(12.1)$ & $29(87.9)$ & & \\
\hline \multicolumn{5}{|c|}{$\begin{array}{l}\text { Use of other antibiotics for } \\
\text { COVID-19, } \mathrm{n}(\%)\end{array}$} \\
\hline Yes & $3(14.3)$ & $18(85.7)$ & $2.5(0.23-26.6)$ & $0.61^{\mathrm{a}}$ \\
\hline No & $1(6.2)$ & $15(93.8)$ & & \\
\hline \multicolumn{5}{|c|}{ No treatment for COVID-19, n (\%) } \\
\hline Yes & $0(0)$ & $9(100)$ & - & $0.55^{\mathrm{a}}$ \\
\hline No & $4(14.3)$ & $24(85.7)$ & & \\
\hline \multicolumn{5}{|c|}{$\begin{array}{l}\text { IV Immunoglobulins use (without } \\
\text { HCQ or AZM), } n(\%)(n=16)\end{array}$} \\
\hline Yes & $0(0)$ & $7(100)$ & - & $1.00^{\mathrm{a}}$ \\
\hline No & $1(11.1)$ & $8(88.9)$ & & \\
\hline \multicolumn{5}{|c|}{$\begin{array}{l}\text { IV Immunoglobulins and } \\
\text { corticotherapy use (without HCQ or } \\
\text { AZM), n }(\%)(n=16)\end{array}$} \\
\hline Yes & $0(0)$ & $4(100)$ & - & $1.00^{\mathrm{a}}$ \\
\hline No & $1(8.3)$ & $11(91.7)$ & & \\
\hline
\end{tabular}

${ }^{a}$ Fisher's Exact Test. Legend: AZM = azithromycin, HCQ = hydroxychloroquine, IV = intravenous. 


\section{Table 3. Ventilatory support outcomes analyzed, length of hospital stay and mortality.}

\begin{tabular}{|c|c|c|c|c|}
\hline Sample & $\begin{array}{l}\text { Gender/ } \\
\text { Age }\end{array}$ & $\begin{array}{l}\text { Treatment for } \\
\text { COVID-19 }\end{array}$ & Treatment for MG & Evolution and LOS \\
\hline
\end{tabular}

\begin{tabular}{|c|c|c|c|c|c|}
\hline $\begin{array}{l}\text { Aksoy \& } \\
\text { Oztutgan (2020) }\end{array}$ & 1 & $\mathrm{~F} / 42$ years & $\begin{array}{c}\text { Favipiravir, } \\
\text { meropenem, } \\
\text { oseltamivir, HCQ } \\
(400 \mathrm{mg} \text { twice daily } \\
\text { on the first day, then } \\
200 \mathrm{mg} \text { twice daily) } \\
\text { and subcutaneous } \\
\text { low molecular weight } \\
\text { heparin. HCQ stopped } \\
\text { on day } 5 \text {. }\end{array}$ & $\begin{array}{c}\text { Pyridostigmine } 240 \mathrm{mg} \\
\text { daily. Methylprednisolone } \\
40 \mathrm{mg} \text { daily, added on } \\
\text { day } 5 .\end{array}$ & $\begin{array}{c}\text { Received VS } \\
\text { after immediate } \\
\text { healing with PLEX. } \\
\text { Discharged on } 22^{\text {nd }} \\
\text { day. }\end{array}$ \\
\hline \multirow[t]{4}{*}{$\begin{array}{l}\text { Anand et al. } \\
(2020)\end{array}$} & 2 & F/ 42 years & $\begin{array}{l}\text { No treatment for } \\
\text { COVID- } 19 .\end{array}$ & $\begin{array}{l}\text { Prednisone } 20 \mathrm{mg} \text { every } \\
\text { day and IVIg } 2 \mathrm{~g} / \mathrm{kg} \text {. }\end{array}$ & $\begin{array}{l}\text { Patient did not } \\
\text { need VS. She was } \\
\text { discharged on the } \\
5^{\text {th }} \text { day. }\end{array}$ \\
\hline & 3 & $\mathrm{~F} / 64$ years & $\begin{array}{l}\text { No treatment for } \\
\text { COVID-19. }\end{array}$ & $\begin{array}{c}\text { MMF } 750 \mathrm{mg} \text { twice } \\
\text { daily, prednisone } 15 \mathrm{mg} \\
\text { every day. MMF was } \\
\text { discontinued } 1 \text { week after } \\
\text { discharge. }\end{array}$ & $\begin{array}{l}\text { Patient did not } \\
\text { need VS and she } \\
\text { was discharged } \\
\text { on the } 9^{\text {th }} \text { day of } \\
\text { hospitalization. }\end{array}$ \\
\hline & 4 & F/ 90 years & $\begin{array}{l}\text { HCQ } 400 \mathrm{mg} \text { twice } \\
\text { daily for } 1 \text { day, and } \\
200 \mathrm{mg} \text { twice daily } \\
\text { for } 4 \text { days; AZM } 500 \\
\text { mg for } 5 \text { days and } \\
\text { ceftriaxone } 1 \mathrm{~g} \text { for } \\
5 \text { days. }\end{array}$ & $\begin{array}{l}\text { MMF discontinued; } \\
\text { prednisone } 25 \mathrm{mg} \text { for } 6 \\
\text { days, then daily; IVIg } \\
\text { continued. }\end{array}$ & $\begin{array}{l}\text { The patient received } \\
\text { VS from the } 10^{\text {th }} \\
\text { to the } 17^{\text {th }} \text { day. She } \\
\text { stayed in the ICU } \\
\text { for } 17 \text { days and then } \\
\text { She was referred to } \\
\text { the ward. }\end{array}$ \\
\hline & 5 & $\begin{array}{l}\mathrm{M} / 57 \\
\text { years }\end{array}$ & $\begin{array}{l}\text { HCQ } 400 \mathrm{mg} \text { twice } \\
\text { daily for } 1 \text { day, } 200 \\
\text { mg twice daily after } \\
\text { day 1; AZM } 500 \mathrm{mg} . \\
\text { AZA } 50 \mathrm{mg} \text { daily. }\end{array}$ & AZA $50 \mathrm{mg}$ daily. & $\begin{array}{l}\text { Patient received IV. } \\
\text { Discharged on the } \\
9^{\text {th }} \text { day after using } \\
\text { tocilizumab } 300 \mathrm{mg} \\
\text { in a single dose. }\end{array}$ \\
\hline
\end{tabular}

HCQ $400 \mathrm{mg}$; AZM on the first day, followed by $250 \mathrm{mg}$ daily for 4 days; ceftriaxone $2 \mathrm{~g}$ for 2 days, and $1 \mathrm{~g}$ three times a day for 3 days.
MMF discontinued, resumed on the $11^{\text {th }}$ day of hospitalization. Prednisone $10 \mathrm{mg}$ for 9 days, then by $5 \mathrm{mg}$ every day. The patient was intubated on the first day of hospitalization and remained on mechanical ventilation after $35^{\text {th }}$ day. There was no further data in the article.
The patient was intubated on the first day of hospitalization and remained on mechanical ventilation after $35^{\text {th }}$ day. There was no further data in the article on the patient's evolution. 


\begin{tabular}{|c|c|c|c|c|c|}
\hline \multirow[t]{15}{*}{$\begin{array}{l}\text { Camelo-Filho et } \\
\text { al. (2020) }\end{array}$} & 7 & NA & $\begin{array}{l}\text { AZM, cefuroxime; } \\
\text { oseltamivir, amikacin, } \\
\text { and teicoplanin. }\end{array}$ & Prednisone $20 \mathrm{mg}$. & $\begin{array}{l}\text { Patient received IVS. } \\
\text { He died after } 13 \text { days } \\
\text { in hospital. }\end{array}$ \\
\hline & 8 & NA & $\begin{array}{c}\text { Piperacilline/ } \\
\text { tazobactame, } \\
\text { ceftriaxone, and AZM. }\end{array}$ & Prednisone $30 \mathrm{mg}$. & $\begin{array}{l}\text { Patient died after } 9 \\
\text { days in hospital. }\end{array}$ \\
\hline & 9 & NA & Ceftriaxone. & $\begin{array}{c}\text { Prednisone and AZA } \\
\text { continued, IVIg } 2 \mathrm{~g} / \mathrm{kg} \\
\text { added. }\end{array}$ & $\begin{array}{l}\text { The Patient was } \\
\text { hospitalized for } 8 \\
\text { days and did not } \\
\text { require mechanical } \\
\text { ventilation. }\end{array}$ \\
\hline & 10 & NA & Ceftriaxone. & $\begin{array}{l}\text { Adding prednisone dose to } \\
40 \mathrm{mg} / \text { day, methotrexate } \\
\text { was maintained. }\end{array}$ & $\begin{array}{l}18 \text { days hospital stay. } \\
\text { Patient remained } \\
\text { hospitalized. }\end{array}$ \\
\hline & 11 & NA & $\begin{array}{l}\text { Ceftriaxone, } \\
\text { oseltamivir, } \\
\text { meropenem, colistin, } \\
\text { and linezolid. }\end{array}$ & $\begin{array}{l}\text { Prednisone was } \\
\text { maintained; } \\
\text { discontinuation of } \\
\text { methotrexate. }\end{array}$ & $\begin{array}{l}\text { Patient received IVS, } \\
\text { and after } 29 \text { days } \\
\text { of hospitalization, } \\
\text { remained hospitalized } \\
\text { at the end of the } \\
\text { study. }\end{array}$ \\
\hline & 12 & NA & $\begin{array}{c}\text { Clarithromycin, } \\
\text { ceftriaxone, AZM, and } \\
\text { oseltamivir. }\end{array}$ & None reported. & $\begin{array}{l}7 \text { days of } \\
\text { hospitalization with } \\
\text { IVS. Died. }\end{array}$ \\
\hline & 13 & NA & $\begin{array}{l}\text { Ceftriaxone, AZM, } \\
\text { piperacilline/ } \\
\text { tazobactame, and } \\
\text { meropenem. }\end{array}$ & $\begin{array}{l}\text { Up the prednisone, } 5 \\
\text { PLEX sessions added; } \\
\text { cyclosporine suspended. }\end{array}$ & $\begin{array}{c}\text { Patient was } \\
\text { hospitalized for } 42 \\
\text { days and needed IVS. }\end{array}$ \\
\hline & 14 & NA & $\begin{array}{c}\text { Piperacilline/ } \\
\text { tazobactame, } \\
\text { ceftrixone, and AZM. }\end{array}$ & $\begin{array}{l}\text { Up prednisone, } 4 \text { PLEX } \\
\text { sessions added; AZA } \\
\text { suspended. }\end{array}$ & $\begin{array}{l}\text { Patient needed IVS. } \\
24 \text { days hospitalized. }\end{array}$ \\
\hline & 15 & NA & Ceftriaxone. & $\begin{array}{l}\text { Prednisone continued, } 5 \\
\text { PLEX sessions added and } \\
\text { AZA suspended. }\end{array}$ & $\begin{array}{l}\text { Patient needed IVS. } \\
20 \text { days hospitalized. }\end{array}$ \\
\hline & 16 & NA & Ceftriaxone and AZM. & Held 5 PLEX sessions. & $\begin{array}{l}\text { Patient remained } \\
\text { hospitalized for } 28 \\
\text { days, } 16 \text { in the ICU. } \\
\text { IVS needed. }\end{array}$ \\
\hline & 17 & NA & AZM. & $\begin{array}{l}\text { Prednisone } 20 \mathrm{mg} \text { and } \\
\text { AZA } 150 \mathrm{mg} .\end{array}$ & $\begin{array}{c}3 \text { days of } \\
\text { hospitalization } \\
\text { without VS. }\end{array}$ \\
\hline & 18 & NA & $\begin{array}{l}\text { Ceftriaxone, } \\
\text { clarithromycin, } \\
\text { meropenem and } \\
\text { vancomycin. }\end{array}$ & $\begin{array}{l}\text { Up Prednisone, dose not } \\
\text { reported. }\end{array}$ & $\begin{array}{l}\text { Hospitalized for } 16 \\
\text { days, } 15 \text { in the ICU. } \\
\text { The patient needed } \\
\text { VS and died. }\end{array}$ \\
\hline & 19 & NA & Ceftriaxone and AZM. & $\begin{array}{l}\text { Prednisone } 60 \mathrm{mg} \text { and } \\
\text { AZA suspended. }\end{array}$ & $\begin{array}{l}\text { Hospital discharge } \\
\text { after } 8 \text { days, } \\
\text { without the need } \\
\text { for mechanical } \\
\text { ventilation. }\end{array}$ \\
\hline & 20 & NA & $\begin{array}{c}\text { AZM, ceftriaxone, } \\
\text { meropenem, linezolid, } \\
\text { amikacyn, polymyxin } \\
\text { B. }\end{array}$ & $\begin{array}{l}\text { Up prednisone, dose not } \\
\text { reported. }\end{array}$ & $\begin{array}{l}\text { Hospital discharge } \\
\text { after } 42 \text { days of total } \\
\text { hospitalization ( } 32 \\
\text { days in the ICU) - } \\
\text { requiring IVS. }\end{array}$ \\
\hline & 21 & NA & Ceftriaxone. & $\begin{array}{l}\text { Increased prednisone, dose } \\
\text { not reported. }\end{array}$ & $\begin{array}{l}\text { Hospital discharge } \\
\text { after } 22 \text { days of total } \\
\text { hospitalization (17 } \\
\text { days in the ICU) - } \\
\text { requiring IVS. }\end{array}$ \\
\hline
\end{tabular}




\begin{tabular}{|c|c|c|c|c|c|}
\hline $\begin{array}{l}\text { Delly et al. } \\
(2020)\end{array}$ & 22 & $\mathrm{~F} / 56$ years & $\begin{array}{c}\text { Vancomycin, } \\
\text { cefepime, AZM } \\
\text { initially. Antibiotics } \\
\text { were discontinued and } \\
\text { HCQ } 200 \text { mg per day } \\
\text { was started. }\end{array}$ & $\begin{array}{c}\text { Prednisone } 40 \mathrm{mg} \\
\text { twice daily, on day } 3 \text {, } \\
\text { pyridostigmine and IVIg } \\
\text { started } 400 \mathrm{mg} / \mathrm{kg} \text { for } \\
5 \text { days. }\end{array}$ & $\begin{array}{l}\text { Patient needed } \\
\text { IVS. She healed } \\
\text { after } 17 \text { days of } \\
\text { hospitalization. } \\
\text { She was waiting } \\
\text { for a subacute } \\
\text { rehabilitation facility. }\end{array}$ \\
\hline
\end{tabular}

\begin{tabular}{|c|c|c|c|c|c|}
\hline $\begin{array}{l}\text { Hofstadt-van Oy } \\
\text { et al. }(2021)\end{array}$ & 23 & $\begin{array}{l}\mathrm{M} / 62 \\
\text { years }\end{array}$ & $\begin{array}{l}\text { Dexamethasone, } \\
\text { heparin, piperacilline/ } \\
\text { tazobactame. }\end{array}$ & $\begin{array}{c}30 \mathrm{~g} \text { intravenous } \\
\text { immunoglobulins } \\
\text { (IVIg) for five days and } \\
\text { pyridostigmine. } \\
\text { PLEX ( } 6 \text { cycles } 3 \text { times } \\
\text { weekly), rituximab } 1000 \\
\text { mg and eculizumab } \\
\text { (900 mg weekly for } \\
4 \text { weeks, followed by } \\
1200 \text { mg every } 2 \text { weeks). }\end{array}$ & $\begin{array}{l}\text { Patient needed } \\
\text { IVS. Tracheostomy } \\
\text { was removed after } \\
\text { the second dose of } \\
\text { eculizumab. Bulbar } \\
\text { symptoms were } \\
\text { completely resolved } \\
10 \text { weeks after the } \\
\text { start of eculizumab. }\end{array}$ \\
\hline
\end{tabular}

\begin{tabular}{|c|c|c|c|}
\hline $\begin{array}{l}\text { Hübers et al. } \\
(2020)\end{array}$ & 24 & $\mathrm{~F} / 36$ years & $\begin{array}{c}\text { No treatment for } \\
\text { COVID- } 19 .\end{array}$ \\
\hline
\end{tabular}

IVIg for 5 days after worsening of $\mathrm{MG}$ symptoms; break from using AZA $50 \mathrm{mg}$ and pyridostigmine $60 \mathrm{mg}$.

o treatment for

$25 \quad \mathrm{~F} / 36$ years COVID-19.
26

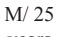

AZM and piperacillin/ tazobactam.

No treatment for COVID-19.

27

M/ 55

years

HCQ for 5 days; AZM avoided; AZA dose

Kushlaf (2020) $\quad 28 \quad$ F/ 66 years

adjusted according

to the patient's renal status.

\section{0 days of}

hospitalization; without VS.
Pyridostigmine $60 \mathrm{mg}$ four to five times a day, prednisone $25 \mathrm{mg}$ per day and subcutaneous immunoglobulins $12 \mathrm{~g}$ every 4 days.

$$
\text { every } 4 \text { days. }
$$

Pyridostigmine $60 \mathrm{mg}$ three times a day.

Patient received IVS. Intubated on the second day and extubated on the $7^{\text {th }}$; hospitalized for 9 days.

Patient received IVS. Tracheostomy removed after 9 weeks.

Hospitalized for 13 days, without receiving VS.

IVS. Extubated IVIg $1 \mathrm{~g} / \mathrm{kg}$ daily for $2 \quad$ after 17 days consecutive days, and and discharged tocilizumab. for hospital rehabilitation.

\begin{tabular}{|c|c|c|c|c|c|}
\hline \multirow[t]{3}{*}{$\begin{array}{l}\text { Octaviana et al. } \\
(2021)\end{array}$} & 29 & $\mathrm{~F} / 25$ years & $\begin{array}{c}\text { Vitamin, NAC, } \\
\text { ceftriaxone, AZM } \\
\text { were discontinued. }\end{array}$ & Pyridostigmine. & $\begin{array}{c}\text { The patient needed } \\
\text { VS and healed after } 5 \\
\text { days of } \\
\text { hospitalization. }\end{array}$ \\
\hline & 30 & F/ 42 years & HCQ and NAC. & MMF and pyridostigmine. & $\begin{array}{l}14 \text { days of } \\
\text { hospitalization } \\
\text { without VS. }\end{array}$ \\
\hline & 31 & $\begin{array}{l}\text { M/ } 49 \\
\text { years }\end{array}$ & $\begin{array}{l}\text { AZM } 500 \mathrm{mg} \text { daily } \\
\text { for } 5 \text { consecutive } \\
\text { days, vitamin C } 3000 \\
\text { mg once daily and } \\
\text { paracetamol } 1500 \mathrm{mg} \\
\text { once daily. }\end{array}$ & $\begin{array}{l}\text { Pyridostigmine } 180 \mathrm{mg} \\
\text { every day and AZA } 100 \\
\text { mg once daily. }\end{array}$ & $\begin{array}{c}\text { The patient needed } \\
\text { VS and healed after } \\
14 \text { days of } \\
\text { hospitalization. }\end{array}$ \\
\hline
\end{tabular}




\begin{tabular}{|c|c|c|c|c|c|}
\hline $\begin{array}{l}\text { Phillip \& } \\
\text { Prerana (2020) }\end{array}$ & 32 & $\begin{array}{l}\mathrm{M} / 70 \\
\text { years }\end{array}$ & $\begin{array}{c}\text { Hydrocortisone } \\
\text { sodium succinate } \\
100 \mathrm{mg} .\end{array}$ & PLEX. & $\begin{array}{l}\text { Patient received } \\
\text { IVS. Extubated after } \\
\text { steroid therapy and } \\
\text { PLEX on } 5^{\text {th }} \text { day. }\end{array}$ \\
\hline $\begin{array}{l}\text { Ramaswamy \& } \\
\text { Govindarajan } \\
(2020)\end{array}$ & 33 & $\mathrm{~F} / 42$ years & $\begin{array}{l}\text { No treatment for } \\
\text { COVID-19. }\end{array}$ & $\begin{array}{c}\text { Pyridostigmine } 30 \mathrm{mg} \\
\text { three times a day. }\end{array}$ & $\begin{array}{l}\text { Quarantine at home } \\
\text { for } 14 \text { days. She did } \\
\text { not need VS. }\end{array}$ \\
\hline \multirow[t]{3}{*}{$\begin{array}{l}\text { Rein et al. } \\
(2020)\end{array}$} & 34 & $\mathrm{~F} / 38$ years & $\begin{array}{l}\text { HCQ } 600 \mathrm{mg} \text { twice } \\
\text { daily, after } 200 \mathrm{mg} \\
\text { three times daily for } \\
\text { additional } 9 \text { days; } \\
\text { lopinavir } 400 \mathrm{mg} \text { twice } \\
\text { daily and ritonavir } \\
100 \mathrm{mg} \text { twice daily } \\
\text { for } 10 \text { days. AZM was } \\
\text { avoided. }\end{array}$ & $\begin{array}{c}\text { IVIg, prednisone } 25 \\
\text { mg once daily and } \\
\text { pyridostigmine } 60 \mathrm{mg} \\
5 \text { times daily. After } \\
\text { exacerbation, she received } \\
\text { IVIg } 2 \mathrm{~g} / \mathrm{kg} \text { for } 5 \text { days and } \\
\text { the prednisone dosage } \\
\text { was titrated up to } 60 \mathrm{mg} \\
\text { per day. }\end{array}$ & $\begin{array}{l}\text { Patient received } \\
\text { VS. After } 10 \text { days } \\
\text { of hospitalization, } \\
\text { and was discharged } \\
\text { with minimal } \\
\text { residual symptoms of } \\
\text { myasthenia. }\end{array}$ \\
\hline & 35 & $\mathrm{~F} / 42$ years & $\begin{array}{l}\text { No treatment for } \\
\text { COVID-19. }\end{array}$ & $\begin{array}{l}\text { IVIg monthly, prednisone } \\
10 \text { mg once daily, and } \\
\text { pyridostigmine. }\end{array}$ & $\begin{array}{l}\text { Home recovery } \\
\text { without VS. }\end{array}$ \\
\hline & 36 & $\begin{array}{l}\mathrm{M} / 66 \\
\text { years }\end{array}$ & $\begin{array}{l}\text { No treatment for } \\
\text { COVID-19. }\end{array}$ & $\begin{array}{l}\text { Prednisone and PLEX was } \\
\text { replaced by IVIg }\end{array}$ & $\begin{array}{l}\text { Patient did not } \\
\text { receive VS. The } \\
\text { symptoms of COVID } \\
19 \text { solved in } 2 \text { days. }\end{array}$ \\
\hline $\begin{array}{l}\text { Salik et al. } \\
(2020)\end{array}$ & 37 & $\begin{array}{l}\mathrm{M} / 80 \\
\text { years }\end{array}$ & $\begin{array}{c}\text { AZM for } 4 \text { days and } \\
\text { HCQ for } 7 \text { days. AZA } \\
\text { performed. HCQ was } \\
\text { removed after patient's } \\
\text { reintubation. }\end{array}$ & $\begin{array}{l}\text { Pyridostigmine, then } 5 \\
\text { days of daily IVIg, after } \\
\text { myasthenic crisis and } \\
\text { reintubation. }\end{array}$ & $\begin{array}{l}\text { Patient received IVIg } \\
\text { for } 6 \text { days reintubed } \\
\text { after aspiration. HCQ } \\
\text { discontinued. He } \\
\text { underwent an open } \\
\text { surgical tracheostomy } \\
\text { on the } 20^{\text {th }} \text { day. }\end{array}$ \\
\hline $\begin{array}{l}\text { Singh \& } \\
\text { Govindarajan } \\
(2020)\end{array}$ & 38 & $\mathrm{~F} / 36$ years & $\begin{array}{l}\text { No treatment for } \\
\text { COVID- } 19 \text {. }\end{array}$ & $\begin{array}{l}\text { Stopped pyridostigmine } \\
\text { and oral prednisone; } \\
\text { MMF continuity. PLEX } \\
\text { performed, } 5 \text { changes } \\
\text { every two days during the } \\
14 \text { days of intubation. }\end{array}$ & $\begin{array}{c}\text { She remained in the } \\
\text { hospital for another } 7 \\
\text { days after extubation } \\
\text { and was discharged, } \\
\text { resuming the home } \\
\text { dose of prednisone } \\
\text { ( } 25 \mathrm{mg} \text { daily) and } \\
\text { MMF (1000mg twice } \\
\text { daily). }\end{array}$ \\
\hline $\begin{array}{l}\text { Sriwastava et al } \\
(2020)\end{array}$ & 39 & $\mathrm{~F} / 65$ years & $\begin{array}{l}\text { Ceftriaxone, AZM, } \\
\text { 1-unit convalescent } \\
\text { plasma, she was } \\
\text { placed in the prone } \\
\text { position for } 1 \mathrm{~h} \text { at } \\
\text { a time for } 4 \text { days, } \\
\text { received intravenous } \\
\text { dexamethasone } 6 \\
\text { mg and } 4 \text { doses } \\
\text { of intravenous } \\
\text { dexamethasone. }\end{array}$ & $\begin{array}{l}\text { Pyridostigmine } 60 \mathrm{mg} \\
\text { every } 6 \mathrm{~h} \text { and after } \\
\text { pyridostigmine dosage } \\
\text { was reduced to } 60 \mathrm{mg} \\
\text { every } 8 \mathrm{~h} .\end{array}$ & $\begin{array}{l}\text { Patient received } \\
\text { VS. After } 10 \text { days } \\
\text { of hospitalization, } \\
\text { she was discharged } \\
\text { with minimal } \\
\text { residual symptoms } \\
\text { of myasthenia ocular } \\
\text { and COVID-19. }\end{array}$ \\
\hline
\end{tabular}

$\mathrm{AZA}=$ azathioprine. $\mathrm{AZM}=$ azithromycin. $\mathrm{F}=$ female gender. $\mathrm{HCQ}=$ hydroxychloroquine. $\mathrm{ICU}$ = intensive care unit. IVIg = intravenous immunoglobulin. IVS = invasive ventilatory support. $\mathrm{M}$ $=$ male gender. $\mathrm{MMF}=$ mycophenolate mofetil. $\mathrm{NAC}=\mathrm{N}$-acetylcysteine. $\mathrm{NA}=$ not applicable. $\mathrm{VS}$ $=$ ventilatory support. $\mathrm{LOS}=$ length of hospital stay. 
Table 4. Factors associated with the necessity of ventilatory support in patients with myasthenia gravis infected by SARS-CoV-2

\begin{tabular}{|c|c|c|c|c|}
\hline Variables & $\begin{array}{l}\text { Ventilatory } \\
\text { Support Yes } \\
(\mathrm{n}=26)\end{array}$ & $\begin{array}{l}\text { Ventilatory } \\
\text { Support } \\
\text { No }(n=11)\end{array}$ & RR (CI 95\%) & $\mathrm{p}$ value \\
\hline \multicolumn{5}{|l|}{ Sex, n (\%) } \\
\hline Masculine & $7(77.8)$ & $2(22.2)$ & $1.9(0.75-2.22)$ & $0.65^{\mathrm{a}}$ \\
\hline Feminine & $9(60)$ & $6(40)$ & 1 & \\
\hline \multicolumn{5}{|c|}{ HCQ use, n (\%) } \\
\hline Yes & $8(88.9)$ & $1(11.1)$ & $1.38(0.96-1.98)$ & $0.22^{\mathrm{a}}$ \\
\hline No & $18(64.3)$ & $10(35.7)$ & 1 & \\
\hline \multicolumn{5}{|c|}{ AZM use, n (\%) } \\
\hline Yes & $17(85)$ & $3(15)$ & $1.60(0.98-2.60)$ & $0.33^{\mathrm{b}}$ \\
\hline No & $9(52.9)$ & $8(47.1)$ & 1 & \\
\hline \multicolumn{5}{|c|}{ HCQ + AZM use, $n(\%)$} \\
\hline Yes & $5(100)$ & $0(0)$ & - & $0.29^{\mathrm{a}}$ \\
\hline No & $21(65.6)$ & $11(34.4)$ & & \\
\hline \multicolumn{5}{|c|}{$\begin{array}{l}\text { Use of other Antibiotics, } \\
\mathrm{n}(\%)\end{array}$} \\
\hline Yes & $17(89.5)$ & $2(10.5)$ & $1.78(1.10-2.91)$ & $0.009^{\mathrm{b}}$ \\
\hline No & $9(50)$ & $9(50)$ & 1 & \\
\hline \multicolumn{5}{|c|}{$\begin{array}{l}\text { IV immunoglobulins use } \\
\text { (without HQC or AZM), } \\
\mathrm{n}(\%)(\mathrm{n}=16)\end{array}$} \\
\hline Yes & $2(28.6)$ & $5(71.4)$ & $0.42(0.12-1.51)$ & $0.31^{\mathrm{a}}$ \\
\hline No & $6(66.7)$ & $3(33.3)$ & 1 & \\
\hline \multicolumn{5}{|c|}{$\begin{array}{l}\text { IV immunoglobulins } \\
\text { and corticotherapy use } \\
\text { (without HQC or AZM), } \\
\mathrm{n}(\%)(\mathrm{n}=16)\end{array}$} \\
\hline Yes & $1(25.0)$ & $3(75.0)$ & $0.43(0.07-2.50)$ & $0.56^{\mathrm{a}}$ \\
\hline No & $7(58.3)$ & $5(41.7)$ & 1 & \\
\hline
\end{tabular}

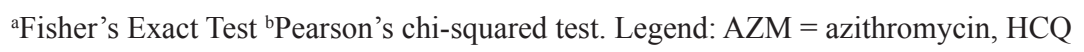
$=$ hydroxychloroquine, $\mathrm{IV}=$ intravenous. 
Table 5. Factors associated with the need for invasive ventilation.

\begin{tabular}{|c|c|c|c|c|}
\hline Variables & $\begin{array}{c}\text { Invasive } \\
\text { Ventilation } \\
\text { Yes } \\
(\mathrm{n}=18)\end{array}$ & $\begin{array}{c}\text { Invasive } \\
\text { Ventilation } \\
\text { No } \\
(\mathrm{n}=21)\end{array}$ & RR (CI 95\%) & $\mathrm{p}$ value \\
\hline \multicolumn{5}{|l|}{ Sex, n (\%) } \\
\hline Masculine & $6(66.7)$ & $3(33.3)$ & $2.50(0.95-6.51)$ & $0.09^{\mathrm{a}}$ \\
\hline Feminine & $4(26.7)$ & $11(73.3)$ & 1 & \\
\hline \multicolumn{5}{|c|}{ HCQ use, n (\%) } \\
\hline Yes & $5(55.6)$ & $4(44.4)$ & $1.63(0.36-7.32)$ & $0.70^{\mathrm{a}}$ \\
\hline No & $13(43.3)$ & $17(56.7)$ & 1 & \\
\hline \multicolumn{5}{|c|}{ AZM use, n (\%) } \\
\hline Yes & $10(47.6)$ & $11(52.4)$ & $1.13(0.32-4.02)$ & $0.84^{\mathrm{b}}$ \\
\hline No & $8(44.4)$ & $10(55.6)$ & 1 & \\
\hline \multicolumn{5}{|c|}{$\begin{array}{l}\mathrm{HCQ}+\mathrm{AZM} \text { use, } \\
\mathrm{n}(\%)\end{array}$} \\
\hline Yes & $4(80)$ & $1(20)$ & $1.94(1.07-3.52)$ & $0.16^{\mathrm{a}}$ \\
\hline No & $14(41.2)$ & $20(58.8)$ & 1 & \\
\hline \multicolumn{5}{|c|}{$\begin{array}{l}\text { Use of other } \\
\text { Antibiotics, n (\%) }\end{array}$} \\
\hline Yes & $11(52.4)$ & $10(47.6)$ & $1.34(0.66-2.73)$ & $0.39^{\mathrm{b}}$ \\
\hline No & $7(38.9)$ & $9(61.1)$ & 1 & \\
\hline \multicolumn{5}{|c|}{$\begin{array}{l}\text { IV immunoglobulins } \\
\text { use (without HQC or } \\
\text { AZM), n }(\%)(n=16)\end{array}$} \\
\hline Yes & $2(28.6)$ & $5(71.4)$ & $0.57(0.16-2.15)$ & $0.62^{\mathrm{a}}$ \\
\hline No & $5(50)$ & $5(50)$ & 1 & \\
\hline \multicolumn{5}{|c|}{$\begin{array}{l}\text { IV immunoglobulins } \\
\text { and corticotherapy } \\
\text { use (without HQC or } \\
\text { AZM), n }(\%)(n=17)\end{array}$} \\
\hline Yes & $1(25.0)$ & $3(75.0)$ & $0.54(0.09-3.26)$ & $0.60^{\mathrm{a}}$ \\
\hline No & $6(46.2)$ & $7(53.8)$ & 1 & \\
\hline
\end{tabular}

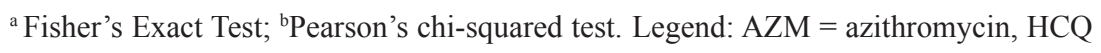
$=$ hydroxychloroquine, IV $=$ intravenous. 
Table 6. The analysis of authors, year of publication, funding and location of the studies included in this review.

\begin{tabular}{|c|c|c|c|}
\hline Authors & Year & Funding & Location \\
\hline Aksoy \& Oztutgan & 2020 & No financing related & Turkey \\
\hline 00Anand et al. & 2020 & No financing related & United States of America \\
\hline Camelo-Filho et al. & 2020 & No financing related & Brazil \\
\hline Delly et al. & 2020 & None & Brazil \\
\hline $\begin{array}{l}\text { Hofstadt-van Oy } \\
\text { et al. }\end{array}$ & 2021 & None & Germany \\
\hline Hübers et al. & 2020 & None & Switzerland \\
\hline Kushlaf & 2020 & No financing related & $\begin{array}{c}\text { Department of Neurology and } \\
\text { Rehabilitation Medicine, University } \\
\text { of Cincinnati, Cincinnati, Ohio } \\
\text { United States of America }\end{array}$ \\
\hline Octaviana et al. & 2021 & $\begin{array}{l}\text { This publication is } \\
\text { funded by Universitas } \\
\text { Indonesia }\end{array}$ & Indonesia \\
\hline Phillip \& Prerana & 2020 & None & United States of America \\
\hline $\begin{array}{l}\text { Ramaswamy \& } \\
\text { Govindarajan }\end{array}$ & 2020 & No financing related & United States of America \\
\hline Rein et al. & 2020 & None & Israel \\
\hline Salik et al. & 2020 & No financing related & United States of America \\
\hline $\begin{array}{l}\text { Singh \& } \\
\text { Govindarajan }\end{array}$ & 2020 & No financing related & United States of America \\
\hline Sriwastava et al. & 2020 & None & $\begin{array}{c}\text { India } \\
\text { United States of America }\end{array}$ \\
\hline
\end{tabular}

\section{DISCUSSION}

The concern regarding patients with Sars-Cov-2 and MG infection centers on two aspects: chronic immunological suppression and respiratory failure due to myasthenic exacerbation (Rein et al., 2020). Establishing the etiology of respiratory failure is very difficult, especially in cases of ventilatory support, requiring neurological consultation (Camelo-Filho et al., 2020). 
In the present study AZM was associated with mortality, need for IVS and elevated LOS compared to the mean. Patients who used HCQ and AZM had almost twice the risk of needing IVS (RR 1.94; 95\% CI 1.07-3.52). The use of other antibiotics in addition to azithromycin for the treatment of COVID-19 was associated with an increase in LOS [20 days (9.5-32) vs 10 days (9-14); $\mathrm{p}=0.01]$. Patients who used hydroxychloroquine (HCQ) and AZM had almost twice the risk of needing IVS (RR 1.94; 95\% CI 1.07-3.52; $\mathrm{p}=0.16$ ). In the study by Camelo-Filho et al. (2020) on patients who died, $75 \%$ used azithromycin and this medication was associated with the clinical worsening of participants in other studies. (Aksoy \& Oztutgan, 2020; CameloFilho et al., 2020; Salik et al., 2020).

The use of azithromycin and hydroxychloroquine may exacerbate COVID-19 and trigger myasthenic attacks (Anand et al., 2020; Gilhus et al., 2018; Jallouli et al., 2011). A retrospective study of 127 participants with autoimmune myasthenia gravis demonstrated worsening of the disease after administration of azithromycin (odds ratio: 1.42) (Abicht et al., 2012; Solé et al., 2020). Therefore, health professionals who work in the coronavirus pandemic must be aware of the risks involved in relation to drugs that worsen clinical conditions and cause respiratory failure in patients with MG.

All of these findings confirm the National Institute of Health guideline, which does not recommend the use of hydroxychloroquine alone or in combination with azithromycin for COVID-19 (NIH, 2020; Fiolet et al., 2020). A clinical trial conducted on non-human primates has shown that neither HCQ nor the combination of HCQ with AZM showed any significant effect on the viral load in the analyzed tissues, did not provide protection against COVID-19 infection and were ineffective in pre-exposure prophylactic treatment (Maisonnasse et al., 2020; Mega et al., 2020).

In the present study both chronic corticosteroid therapy and the use of IVIg were associated with less need for ventilation, mortality and length of hospital stay. Regarding IVIg associated prednisone, there were interesting trends, since patients using IVIg and prednisone had almost half the risk of IVS and shorter hospital stays ( 8 days vs 16.5 days in median). Another important fact is that the mortality rate of $10.3 \%(\mathrm{n}=4 / 39)$ was not associated with immunosuppressive treatment, IVIg or PLEX. However, we recognize the need for further studies with larger samples to draw conclusions based on stronger evidence.

It is important to highlight that immunosuppressive therapy in immunosuppressed patients does not uniformly present a higher complication risk. Patients using chronic immunosuppressants to prevent transplant rejection responded positively to COVID-19 infection, despite their immunocompromised status (Jacob et al., 2020). It is therefore essential that physicians be critical when discontinuing immunosuppressive drugs in the midst of the pandemic. 
Discontinuity of immunosuppressive therapy should only be considered in a patient with Sars-Cov-2 and MG if the symptoms are severe, such as in hospitalization and bacterial infections/sepsis. Immune depletion agents should be avoided in these situations, while AZA and MMF can be continued, since the dosage effects are more persistent, the elimination of the agent is slower, and the reconstruction of the effects takes months. In addition, it is advisable for patients using medications that cause profound lymphopenia to undergo prophylaxis with antivirals in the post-infusion phase (Jacob et al., 2020).

Depending on the infection stage, corticosteroids, such as prednisone, may have adverse effects, initially causing viremia and impairing viral clearance. Later, however, they inhibit the migration of immune cells and the production of chemokines, reducing the cytokine storm. For this reason, the relationship between chronic corticosteroid therapy, COVID-19 and MG has become essential (Rein et al., 2020).

In the study by Camelo-Filho et al. (2020), all patients who did not require VS used prednisone associated with a second immunosuppressive drug. In addition, Delly et al. (2020) reported that a participant in his study who was in IVS showed improvement in the respiratory function after IVIg, being extubated early.

According to Diez et al. (2020), the intravenous immunoglobulins available on the market proved to react against COVID-19 and other viral antigens in vitro. This is because IVIg preparations contain proteins collected from thousands of donors and ameliorate the course of inflammatory autoimmune diseases by blocking Fc-gamma receptors, neutralizing inflammatory cytokines (Baker et al., 2017).

In cases of severe COVID-19, early administration of high-dose IVIg ( $25 \mathrm{~g}$ /day for 5 days), combined with antivirals and methylprednisolone, allows lymphocytosis, reduction of inflammatory markers, resolution of pulmonary disorders and nasal and negative oropharyngeal smear tests a few days after therapy (Cao et al., 2020; Gasparyan et al., 2020; Paez et al, 2020). Since this medication optimizes passive immunity and modulates the inflammatory response, it has great potential and promising effects when used at the onset of clinical deterioration by coronavirus 2 , not when systemic damage has already set in (Cao et al., 2020; Jawhara, 2020; Lin L et al., 2020).

A recent meta-analysis study found that anti-inflammatory agents (corticosteroids, tocilizumab, anakinra and IVIg), convalescent plasma and remdesivir are associated with better prognosis in hospitalized patients with COVID-19. However, there are still no studies with a high level of evidence involving these agents and MG associated with COVID-19 (Kim et al., 2020).

It is important to note that, in relation to plasmapheresis, Aksoy \& Oztutgan (2020) report that a participant in their study received PLEX therapy and did not need IVS due to immediate improvement and speedy discharge. 
Studies have shown that both plasmapheresis and IVIg prevent worsening of lymphocyte count and should be promptly administered to patients with COVID-19 for more effective and safer treatment. This administration must occur before the $14^{\text {th }}$ day, since early initiation is related to better outcomes (Ramtin et al., 2020).

One participant from Anand et al. (2020) who was in IVS was discharged on the $9^{\text {th }}$ day after using tocilizumab ( $300 \mathrm{mg}$ in a single dose). According to a retrospective study, carried out with 21 seriously ill patients by COVID-19, after the use of tocilizumab, an interleukin-6 inhibitor (IL-6), the clinical picture and alterations in the tomography improved immediately. This suggests that the drug may be effective for the treatment of Sars-Cov-2, when there are no contraindications (Xu et al., 2020).

Among the limitations in this article is the fact that prospective primary studies were scarce and the lack of information caused difficulties in establishing a causal link between therapeutic measures and medium and long-term outcomes. In addition, there was no analysis to verify whether the unfavorable course of the condition was associated with other variables, such as age, gender, previous pathological history, and secondary bacterial infections; as well as no correlations with the other medication also used during the patient's hospitalization.

\section{FINAL CONSIDERATIONS}

The literature review presented in this article leads to the conclusion that there is no consensus on the conduct to be taken in patients with MG infected by Sars-Cov-2, since the clinical course is quite varied, probably related to the general health status of patients and their comorbidities. Despite this, there was an association between the use of hydroxychloroquine and azithromycin with a worse prognosis, in concordance with other articles, which also performed this correlation.

For this reason, it is important that health professionals working on the "frontline" coping with the pandemic do not prescribe drugs, like AZM, HCQ and other antibiotics with harmful effects for MG. In addition, IVIg immunotherapy and corticotherapy are recommended for patients who have respiratory complications, without contraindications to the drug. As demonstrated, basic immunosuppressive therapy was not harmful. In short, as myasthenia gravis is a rare disease, more case reports are needed, with reliable statistical analysis, to better understand the implications between MG, SarsCov-2 infection, and drug therapies. 


\section{CONFLICT OF INTEREST.}

There is no conflict of interest to declare.

\section{REFERENCES}

1. Abicht A, Dusl M, Gallenmüller C, Guergueltcheva V, Schara U, Marina AD, Wibbeler E, Almaras S, Mihaylova V, Von Der Hagen M, Huebner A, Chaouch A, Muller JS, Lochmuller H. Congenital myasthenic syndromes: achievements and limitations of phenotype-guided gene-after-gene sequencing in diagnostic practice: a study of 680 patients. Hum Mutat 33: 1474-1484, 2012.

2. Aksoy E, Oztutgan T. COVID-19 Presentation in Association with Myasthenia Gravis: A Case Report and Review of the Literature. Case Rep Infect Dis 2020: 8845844, 2020.

3. Anand P, Slama MC, Kaku M, Ong C, Cervantes-Arslanian AM, Zhou L, David WS, Guidon AC. COVID-19 in patients with myasthenia gravis. Muscle Nerve 62: 254-258, 2020.

4. Baker D, Herrod SS, Alvarez-Gonzalez C, Zalewski L, Albor C, Schmierer K. Both cladribine and alemtuzumab may effect MS via B-cell depletion. Neurol Neuroimmunol Neuroinflamm 4: e360, 2017.

5. Camelo-Filho AE, Silva AM, Estephan EP, Zambon AA, Mendonça RH, Souza PV, Pinto WB, Oliveira AS, Dangoni-Filho I, Pouza AF, Valerio BC, Zanoteli E. Myasthenia Gravis and COVID-19: Clinical Characteristics and Outcomes. Front Neurol 11: 1053, 2020.

6. Cao W, Liu X, Bai T, Fan H, Hong K, Song H, Han Y, Lin L, Ruan L, Li T. High-dose intravenous immunoglobulin as a therapeutic option for deteriorating patients with coronavirus disease 2019. Open Forum Infect Dis 7: ofaa102, 2020.

7. CONITEC. Comissão Nacional de Incorporação de Tecnologias no Sistema Único de Saúde. Protocolo clínico e diretrizes terapêuticas da Miastenia Gravis. Available in: http://conitec. gov.br/images/Consultas/Relatorios/2020/Relatorio_PCDT_Miastenia_Gravis_CP_27_2020. pdf. Accessed in: 28.sept.2020.

8. Coronavirus Resource Center [Internet]. Baltimore: Johns Hopkins Bloomberg School of Public Health. Mortality Analyses. Available from: https://coronavirus.jhu.edu/data/mortality. Accessed at: 28.march 2021.

9. Delly F, Maryam JS, Lisak RP, Zutshi D. Myasthenic crisis in COVID-19. J Neurol Sci 414: 116888, 2020.

10. Díez JM, Romero C, Gajardo R. Currently available intravenous immunoglobulin contains antibodies reacting against severe acute respiratory syndrome coronavirus 2 antigens. Immunotherapy 12: 571-576, 2020.

11. Emanuel EJ, Persad G, Upshur R, Thome B, Parker M, Glickman A, Zhang C, Boyle C, Smith M, Phillips JP. Fair Allocation of Scarce Medical Resources in the Time of Covid-19. N Engl J Med 382: 2049-2055, 2020.

12. Fiolet T, Guihur A, Rebeaud ME, Mulot M, Peiffer-Smadja N, Mahamat-Saleh Y. Effect of hydroxychloroquine with or without azithromycin on the mortality of coronavirus disease 2019 (COVID-19) patients: a systematic review and meta-analysis. Clin Microbiol Infect 27: 19-27, 2020.

13. Gasparyan AY, Misra DP, Yessirkepov M, Zimba O. Perspectives of immune therapy in coronavirus disease 2019. J Korean Med Sci 35: e176, 2020.

14. Gilhus NE, Romi F, Hong Y, Skeie GO. Myasthenia gravis and infectious disease. J Neurol 265: 1251-1258, 2018. 
15. Hofstadt-van Oy U, Stankovic S, Kelbel C, Oswald D, Larrosa-Lombardi S, Barchfeld T, Cleff U. Complement inhibition initiated recovery of a severe myasthenic crisis with COVID-19. $J$ Neurol 2021: 1-4, 2021.

16. Hübers A, Lascano AM, Lalive PH. Management of patients with generalised myasthenia gravis and COVID-19: four case reports. J Neurol Neurosurg Psychiatry 91: 1124-1125, 2020.

17. Ioannidis JP. Infection fatality rate of COVID-19 inferred from seroprevalence data. Bull World Health Organ 99: 19-33F, 2021.

18. Jacob S, Muppidi S, Guidon A, Guptill J, Hehir M, Howard JF, Illa I, Mantegazza R, Murai H, Utsugisawa K, Vissing J, Wiendl H, Nowak RJ. Guidance for the management of myasthenia gravis (MG) and Lambert-Eaton myasthenic syndrome (LEMS) during the COVID-19 pandemic. J Neurol Sci 412: 116803, 2020.

19. Jallouli M, Saadoun D, Eymard B, Leroux G, Haroche J, Huong DL, Gennes De C, Chapelon C, Benveniste O, Wechsler B, Cacoub P, Amoura Z, Piette JC, Costedoat-Chalumeau N. The association of systemic lupus erythematosus and myasthenia gravis: a series of 17 cases, with a special focus on hydroxychloroquine use and a review of the literature. J Neurol 259: 12901297, 2011.

20. Jaretzki A, Barohn RJ, Ernstoff RM, Kaminski HJ, Keesey JC, Penn AS, Sanders DB. Myasthenia gravis: recommendations for clinical research standards. Task Force of the Medical Scientific Advisory Board of the Myasthenia Gravis Foundation of America. Neurology 55: 16-23, 2000.

21. Jawhara S. Could intravenous immunoglobulin collected from recovered coronavirus patients protect against covid-19 and strengthen the immune system of new patients? Int J Mol Sci 21: 2272, 2020.

22. Keller KG, Reangsing C, Schneider JK. Clinical Presentation and Outcomes of Hospitalized Adults with COVID-19: a systematic review. $J$ Adv Nurs 76: 3235-3257, 2020.

23. Kim MS, An MH, Kim WJ, Hwang T. Comparative efficacy and safety of pharmacological interventions for the treatment of COVID-19: A systematic review and network meta-analysis. PLoS Med 17: e1003501, 2020.

24. Kushlaf H. COVID-19 in muscle-specific kinase myasthenia gravis: a case report. Muscle Nerve 1: 1-1, 2020.

25. Lin L, Lu L, Cao W, Li T. Hypothesis for potential pathogenesis of SARS-CoV-2 infection-a review of immune changes in patients with viral pneumonia. Emerg Microbes Infect 9: 727$732,2020$.

26. Maisonnasse P, Guedj J, Contreras V, Behillil S, Solas C, Marlin R, Naninck T, Pizzorno A, Lemaitre J, Gonçalves A, Kahlaoui N, Terrier O, Fang RH, Enouf V, Dereuddre-Bosquet N, Brisebarre A, Touret F, Chapon C, Hoen B, Lina B, Calatrava MR, Van Der Werf S, Lamballerie X, Le Grand R. Hydroxychloroquine use against SARS-CoV-2 infection in nonhuman primates. Nature 585: 584-587, 2020.

27. Mega TA, Feyissa TM, Bosho DD, Goro KK, Negera GZ. The Outcome of Hydroxychloroquine in Patients Treated for COVID-19: Systematic Review and Meta-Analysis. Can Respir J 2020: 33082891, 2020.

28. Moher D, Liberati A, Tetzlaff J, Altman DG. Preferred reporting items for systematic reviews and meta-analyses: the PRISMA statement. BMJ 339: b2535, 2009.

29. National Institutes of Health. 2020. Chloroquine or Hydroxychloroquine With or Without Azithromycin. Available from: https://www.covid19treatmentguidelines.nih.gov/antiviraltherapy/hydroxychloroquine-plus-azithromycin/. Accessed at: 10/12/2020.

30. Octaviana F, Yugo HP, Safri AY, Indrawati LA, Wiratman W, Ayuningtyas T, Hakin M. Case series: COVID-19 in patients with mild to moderate myasthenia gravis in a National Referral Hospital in Indonesia. eNeurologicalSci 23: 100332, 2021. 
30. Paez YM, Bennett JL, Subramanian PS, Pelak VS. Considerations for the Treatment of Inflammatory Neuro-Ophthalmologic Disorders During the COVID-19 Pandemic. $J$ Neuroophthalmol 40: 305-314, 2020.

31. Ramaswamy SB, Govindarajan R. COVID-19 in Refractory Myasthenia Gravis: A Case Report of Successful Outcome. J Neuromuscul Dis 7: 361-364, 2020.

32. Ramtin P, Bobak M, Nima R. Efficacy of Plasmapheresis and Immunoglobulin Replacement Therapy (IVIG) on Patients with COVID-19. SN Compr Clin Med 31: 1-5, 2020.

33. Rein N, Haham N, Orenbuch-Harroch E, Romain M, Argov Z, Vaknin-Dembisnky A, Gotkine M. Description of 3 patients with myasthenia gravis and COVID-19. J Neurol Sci 417: 117053, 2020.

34. Remuzzi A, Remuzzi G. COVID-19 and Italy: what next? Lancet 395: 1225-1228, 2020.

35. Ruiter AM, Verschuuren GM, Tannemaat MR. Fatigue in patients with myasthenia gravis: a systematic review of the literature. Neuromuscul Disord 30: 631-639, 2020.

36. Salik, I, Rodhouse HB, Barst S. Myasthenic crisis in the setting of Coronavirus Disease 2019 (COVID-19). J Clin Anesth 67: 110001, 2020.

37. Santos CM, Pimenta CA, Nobre MR. A estratégia PICO para a construção da pergunta de pesquisa e busca de evidências. Rev Lat Am Enfermagem 15: 508-511, 2007.

38. Schumm F, Wiethölter H, Fateh-Moghadam A. Myasthenie-Syndrom unter CloroquinTherapie. Dtsch Med Wochenschr 106: 1745-1747, 1981.

39. Shea JB, Reeves BC, Wells G, Thuku M, Hamel C, Moran J, Moher D, Tugwell P, Welch V, Kristjansson E, Henry DA. AMSTAR 2: a critical appraisal tool for systematic reviews that include randomized or non-randomised studies of healthcare interventions, or both. BMJ 358: j4008, 2017.

40. Singh S, Govindarajan R. COVID-19 and generalized Myasthenia Gravis exacerbation: a case report. Clin Neurol Neurosurg 196: 1-2, 2020.

41. Solé G, Salort-Campana E, Pereon, Y, Stojkovic T, Wahbi K, Cintas P, Adams D, Laforet P, Tiffreau V, Desguerre I, Pisella LI, Molon A, Attarian S. Guidance for the care of neuromuscular patients during the COVID-19 pandemic outbreak from the French Rare Health Care for Neuromuscular Diseases Network. Rev Neurol (Paris) 176: 507-515, 2020.

42. Sriwastava S, Tandon M, Kataria S, Daimee M, Sultan S. New onset of ocular myasthenia gravis in a patient with COVID-19: a novel case report and literature review. J Neurol 2020: $1-7,2020$.

43. Xu X, Han M, Li T, Sun W, Wang D, Fu B, Zhou Y, Zheng X, Yang Y, Li X, Zhang X, Pan A, Wei H. Effective treatment of severe COVID-19 patients with tocilizumab. Proc Natl Acad Sci USA 117: 10970-10975, 2020. 